University of Nebraska - Lincoln

DigitalCommons@University of Nebraska - Lincoln

USDA National Wildlife Research Center - Staff

Publications

U.S. Department of Agriculture: Animal and Plant Health Inspection Service

May 1993

\title{
Short Courses as Part of Statistical Consulting in a Foreign Setting
}

Richard M. Engeman

Denver Wildlife Research Center, USDA/APHIS/S\&T, s_r100@yahoo.com

Follow this and additional works at: https://digitalcommons.unl.edu/icwdm_usdanwrc

Part of the Environmental Sciences Commons

Engeman, Richard M., "Short Courses as Part of Statistical Consulting in a Foreign Setting" (1993). USDA National Wildlife Research Center - Staff Publications. 166.

https://digitalcommons.unl.edu/icwdm_usdanwrc/166

This Article is brought to you for free and open access by the U.S. Department of Agriculture: Animal and Plant Health Inspection Service at DigitalCommons@University of Nebraska - Lincoln. It has been accepted for inclusion in USDA National Wildlife Research Center - Staff Publications by an authorized administrator of DigitalCommons@University of Nebraska - Lincoln. 
This department publishes articles of interest to statistical practitioners. Innovative applications of known methodology may be suitable, but sizable case studies should be submitted to other journals.
Brief descriptions and illustrations of new developments that are potentially useful in statistical practice are appropriate. Acceptable articles should appeal to a substantial number of practitioners.

\section{Short Courses as Part of Statistical Consulting in a Foreign Setting}

\section{RICHARD M. ENGEMAN*}

\begin{abstract}
It is common for many consulting statisticians to provide statistical training to their clients. The number and depth of potential difficulties that can arise when giving a short course are greatly increased when the course is given in a foreign setting. Recommendations and examples are given for overcoming these difficulties and for efficient presentation of the course material.
\end{abstract}

KEY WORDS: Culture; Foreign language; Training.

Instructing clients and collaborators in the use of statistical concepts and methods is an important component of work for many, if not most, consulting statisticians (e.g., Kirk 1991). This teaching usually occurs as a necessary by-product in the consulting process so that the client can better understand the statistician's rationale. However, it is occasionally elevated to a formal seminar or short course if there are a number of people who would benefit from the instruction. The clients may be well versed in other disciplines while remaining statistically inexperienced.

The statistician is faced with explaining complex ideas without being able to rely on the potentially confusing jargon of our profession to clarify the situation. This problem is greatly magnified in a foreign setting where there may be language barriers and different customs. The statistician must be innovative and resourceful to teach successfully under these circumstances. The purpose of this article is to describe a difficult classroom situation and some of the methods that I used to successfully teach statistical methods and concepts.

\section{THE SCENARIO}

The Denver Wildlife Research Center agreed to work with Moroccan scientists on an experiment that would evaluate and compare the environmental hazards from

\footnotetext{
${ }^{*}$ Richard M. Engeman is a statistician at the Denver Wildlife Research Center, USDA/APHIS/S\&T, Building 16, Denver Federal Center, P.O. Box 25266, Denver, CO 80225-0266.
}

two insecticides commonly used for spraying swarms of locusts. The experiment would include evaluating their effects on populations of birds, small mammals, and insects. Prior to initiation of the field experiment, the Denver Wildlife Research Center was to provide training to the Moroccan scientists in a number of scientific disciplines including: analytical chemistry, radio telemetry, and sampling methods for bird, mammal, and insect populations. The final training session was my session on experimental design. This session was the largest (19 participants) because it applied to the scientists from all of the disciplines covered in the previous training sessions.

Arabic and French are the official languages of Morocco. Fortunately, the scientists in my session all understood English to varying degrees. Many had studied or worked in Germany at one time or another and could speak German better than English. They were a somewhat statistically naive audience, but they each had a relatively high level scientific background. My task was to provide them with a useful background in experimental design and develop, collaboratively with the Moroccan and American scientists, the design for the environmental hazards study. The training was to be accomplished in a one-week session in Agadir, Morocco, using a language (English) with which they were not $100 \%$ comfortable. The remainder of this article describes the methods that I found useful for teaching the course.

\section{INSTRUCTIONAL MATERIALS}

\subsection{Content and Organization}

The presentation of material and the interactions with a class (or a group of clients) when teaching a short course is greatly facilitated if each member of the class has some instructional materials to serve as a guide to the course and as reference materials after the course. The value of these materials increases with the amount of the information that is also presented orally. Naturally, clear and concise prose with a minimum of technical jargon increases the value of these materials to 
the class. It is also helpful in the classroom if the instructional materials are packaged conveniently so that classroom topics are easily followed, with minimal (disruptive) searching to find the correct topic or page. The class is more sharply focused with a greater interest if an effort has been made to customize the contents of the instructional materials for problems and situations with which the class is involved. This will also better facilitate communication and interaction with the instructor. Learning is promoted if the organization of the materials is such that concepts presented early in the course are reiterated while new concepts are added, especially when course topics relate more specifically to the interests of the class.

\subsection{Training Manual Example}

The material presented in the course was contained in, and centered around, a training manual. The strategy used in the training manual was to first introduce general concepts, then take topics with which the class had increasing interest and involvement and relate them to the design concepts. The purpose for this was not only to draw the class deeper and deeper into the subject matter, but also to promote the revisitation of the key concepts many times in different contexts and depths. This strategy for structuring the manual, and the course, was a key for its success.

The training manual is 45 pages long and the contents, listed in Table 1, illustrate how the above strategies were applied for reinforcing general concepts by moving to specific items of interest. The first half of the manual presents the concepts, while the middle three sections use past studies and data sets increasingly specific to the Moroccans' interests to illustrate the concepts. The last two sections and the appendices serve as reference material. Throughout the manual, the explanations accompanying definitions or concepts usually contain examples specifically relating to environmental hazards studies.

The manual begins with a brief explanation of the course objectives in Section 1. Section 2 provides a definition and outline of what experimental design entails and the next three sections expand on concepts and implementation of experimental design.

\section{Table 1. Training Manual Contents}

1. Purpose of Training

2. Experimental Design Concepts

3. Capabilities Needed for Producing a Good Experimental Design

4. Characteristics of a Good Experimental Design

5. Elements in the Design and Execution of an Experiment

6. An Example of a Study to Evaluate Environmental Hazards

7. Some Analytical Results Using Data Collected from Training Exercises

8. Considerations for Designing a Study in Morocco on the Environmental Effects from Pesticides

9. Some General Comments on Common Data Analyses

10. References

Appendix 1. Bird Count Data Analyses

Appendix 2. Insect Data Analyses
These general conceptual sections are followed by one containing an example of a previous study in Senegal where environmental hazards from five insecticide treatments were studied. This related study increased the interest of the students and increased their participation as the design of that study was evaluated relative to the information and concepts previously presented. For example, the topics covered in Section 4, Characteristics of a Good Experimental Design, include:

1. Absence of systematic error

2. Precision

3. Breadth of inferences

4. Simplicity

5. Data analyses

Although the class could relate to these areas, they were still somewhat abstract until they were applied to the Senegal example. This allowed the strengths and weaknesses of that previous study to be readily apparent. Subsequently, the same criteria were applied to each potential design for the Moroccan environmental hazards study (discussed in Section 8). This further elevated class interest and participation while driving the experimental design concepts home.

Section 7 involves analyses and inferences for data that the people in the class had collected during their earlier training sessions and, therefore, covered information from a point of view with which the class could intimately relate (I had received these data one month prior to my session). Following that, Section 8 (as indicated above) gives a skeleton outline for designing the experiments for the environmental hazards study in which the class would participate. This section was purposely left largely blank to allow the class to apply what they had learned so far and contribute to the development of a design for our cooperative study. Sections 9 and 10 involve some general notes on analytical data methods and references. These sections were not covered in detail in class, but were included to give the class some starting points if they wanted more information on analyzing data. Finally, the two appendices contain the computer outputs from the analyses of the data from the previous training sessions. The participants were not expected to have a computing background, but they were interested in seeing how their data were processed.

The training manual was directed specifically towards the area of experimental design with a specific study in mind. Training material content for other courses would differ greatly, but the utility of proceeding from general concepts to applications customized for the class still would serve to increase class interest and involvement, and hence, learning.

\section{CLASSROOM INTERACTION WITH LANGUAGE AND CULTURAL BARRIERS}

As with most consulting sessions, the success of a short course or training session is affected by the interpersonal skills of the instructor in addition to his or 
her technical background (and the backgrounds of the class members). Much of the considerable attention recently given to interpersonal skills for a consulting session (e.g., Boen and Zahn 1982; Zahn and Isenberg 1983) applies to the short course situation. Presenting a short course to a class from a different culture and language places an even higher premium on recognizing the importance of interpersonal skills. This particular classroom situation required the recognition of several factors crucial to successful communication.

\subsection{Basic Methods for Handling the Language Barrier}

The most obvious factor to potentially influence communication in a foreign setting is a language barrier. Acknowledging and accepting this as the situation going into the training session will make it a point of fact that must be dealt with, rather than a source of frustration. However, clear communication is required to present the course material adequately. Teaching techniques for accomplishing clear communication should incorporate the obvious, such as speaking slowly, with distinct pronunciation, while using more common English words with fewer syllables than usually found in math/science terms. Speaking in this manner requires discipline, including anticipation of upcoming phrases by mentally preparing synonyms for words that the class would have difficulty understanding.

\subsection{Other Methods for Overcoming Language Barriers}

Creative techniques beyond the above can facilitate communication greatly. The instructor should be prepared to use knowledge of whatever languages he has to find some common ground with the class. As an example, I cannot speak French, the second language of Morocco, but I have an excellent grasp of Spanish and a reasonable understanding of German. Despite Morocco's geographic situation across the straits of Gibraltar from Spain, Spanish was of no use for providing a common language nor common words with the class. However, about half or more of the class had worked or studied in Germany at one time or another. For them, German was more readily understood than English and it was common for individuals to impulsively speak or ask questions in German (even while grasping for an English phrase). I would promote communication by coaxing these individuals to continue by saying "Ich verstehe" (German for "I understand").

If the instructor demonstrates his or her commitment to communication, the class will probably reciprocate. Searching for words in common-ground languages (above) beyond the first language of the instructor or class is one such demonstration. Another such demonstration is to quickly learn each person's name and pronounce it correctly, which is not an easy task if the instructor is not familiar with their native language and pronunciations. Accomplishing this will build further rapport and confidence between the class and instructor.
The class may produce its own methods for clarifying communication. The instructor should be open to the additional help, even if he or she doesn't initiate, control, or understand it. My class frequently would hold a conference among themselves (in Arabic), sometimes intense, to resolve a question or to clarify a word or concept. This usually resulted in one of two outcomes. They would resolve the question themselves or they would define the question very well so that I could easily decide how to clear it up. These "hurdles" were very useful for the general understanding of the class, but also required much patience on my part because I could understand none of the gist of the conversation.

Another useful method for communication is to teach using analogies with which the class is familiar. This can be especially useful for teaching statistical terms. As an example in experimental design, to get across the concept of controlling sources of variability, I discussed an example of playing a radio and attempting to hear music in the face of background static. Confounding was illustrated in a similar manner by considering a comparison among the effects on flavor when using water, olive oil, and vegetable oil for cooking. Suppose some water is added to a fish dish, olive oil to a chicken dish, and vegetable oil to a lamb dish. Subsequently, it is found that the three meals taste different. It is easy for the class to understand that the differences could have occurred because the meals were inherently different and do not allow a valid test for the effects of water, olive oil, and vegetable oil. Saving and retrieving computer files were explained by analogy to using books on a bookshelf. One cannot use a book unless it is retrieved from a bookshelf. When finished reading, one must return the book to the shelf (saved) so that it is available to be read again later, rather than throwing it away. The instructor should be aware that different cultures may warm to different analogies. A little familiarity with the culture will help devise useful analogies.

\subsection{Cultural Differences}

There are also cultural considerations beyond language that come into play when teaching a foreign short course. The class will recognize that the instructor comes from a different background, but it promotes a more comfortable interaction if the instructor is accommodating and respectful of cultural differences. As with language, the instructor should accept that different cultures have different ways of doing things, and teach within those constraints. If I had not expected the classroom situations to reflect these differences, then I would have found some procedures to be very disruptive for teaching. For example, mint tea was served at exactly the same time each day without regard to what the class was involved in at that time. Also, lunch breaks averaged close to three hours. Instead of finding the breaks in the flow frustrating, I used them to become better acquainted with the members of the class and to improve communication. 
Being social and thus not appearing to be condescending nor holding the class members at arm's length is an important factor in establishing rapport and learning about their customs and individual talents. This can help the instructor gain insight and information that can prove useful for making the course more interesting and relevant. Most courses have lunch breaks, which can provide the instructor an opportunity to learn about customs and become more acquainted with the members of the class, especially if he or she accompanies members of the class to places they would normally eat (rather than heading to the nearest tourist eatery). In my situation I was able to accumulate information on customs, culture, and individual abilities that also was valuable for producing a practical experimental design to which the Moroccans would commit.

\subsection{Total Involvement}

As with traditional consulting sessions, teaching a short course to a group works best if the philosophy of "total involvement" (e.g., Marquardt 1979) is adopted. The instructor is part of the classroom team with the joint goal of increasing the knowledge and skills of the class so that they can better perform their jobs. This requires the instructor to also learn from the class such things as what their jobs are and why they expect the information being presented to benefit them. This promotes participation and learning as well as a long-term commitment to the objectives of the course (the environmental hazards study in my case). As examples, I found in my Morocco session that it was valuable to the teaching process and to the experimental design process to recognize the status of the class members as scientists and the importance of their contributions for developing the experimental design for the environmental hazards study. When interacting with the class to develop the experimental design, I tried to present my thoughts not as absolutes but rather as "what if," thus avoiding appearing condescending. This drew the class deeper into the topic, but still allowed me to control the direction and, if necessary, the outcome of discussions on experimental design.

\section{CONCLUSIONS}

The presentation of a short course on experimental design in Morocco helped to define methods that gen- erally are useful for teaching short courses or training sessions in foreign settings. Since that time, additional experience delivering training sessions in other foreign settings has reaffirmed the value of these methods. Following is a summary of some general suggestions for presenting a short course in a foreign country.

1. Prepare relevant instructional materials for each member of the class. They should be written using words that are more easily understood by people whose first language is not English. Statistical jargon should be explained in as simple terms as possible or avoided. New information should be presented so that it reinforces the understanding of information already presented. It pays to have extra copies of the instructional materials available.

2. Relate course topics and materials, especially examples, to the areas of interest of the class members. This is particularly effective if the examples used pertain to a specific project with which the class is involved.

3. Demonstrate your commitment to having the class members understand what is being presented. This begins with providing clear communication by using the speaking methods discussed earlier. Other creative methods are also helpful, such as establishing what other languages may provide a common ground for communication and finding alternate ways of explaining words or concepts.

4. Be accommodating and respectful of the backgrounds and attitudes of the class members (this is universal, not for just dealing with foreign clients). You do not have to adopt their customs, but you can understand them and use them to establish a better rapport with the class. This will lead to greater success in the presentation and acceptance of the course topics.

[Received April 1991. Revised March 1992.]

\section{REFERENCES}

Boen, J. R., and Zahn, D. A. (1982), The Human Side of Statistical Consulting, Belmont, CA: Lifetime Learning Publications.

Kirk, R. E. (1991), "Statistical Consulting in a University: Dealing With People and Other Challenges," The American Statistician, $45,28-34$.

Marquardt, D. W. (1979), "Statistical Consulting in Industry," The American Statistician, 33, 102-107.

Zahn, D. A., and Isenberg, D. J. (1983), "Nonstatistical Aspects of Statistical Consulting," The American Statistician, 37, 297-302. 